\title{
Modeling of photoluminescence in laser-based lighting systems
}

Elisavet Chatzizyrli, Nadine Tinne, Roland Lachmayer, Jörg Neumann, Dietmar Kracht

Elisavet Chatzizyrli, Nadine Tinne, Roland Lachmayer, Jörg Neumann, Dietmar Kracht, "Modeling of photoluminescence in laser-based lighting systems," Proc. SPIE 10603, Photonics, Devices, and Systems VII, 1060318 (1 December 2017); doi: 10.1117/12.2292735

SPIE. Event: Photonics Prague 2017, 2017, Prague, Czech Republic 


\title{
Modeling of photoluminescense in laser-based lighting systems
}

\author{
Elisavet Chatzizyrli*a,b, Nadine Tinne, ${ }^{\mathrm{a}, \mathrm{b}}$, Roland Lachmayer ${ }^{\mathrm{a}, \mathrm{b}, \mathrm{c}}$, Jörg Neumann ${ }^{\mathrm{b}}$, Dietmar Kracht ${ }^{\mathrm{b}}$ \\ ${ }^{a}$ Tailored Light, PhD program, Gottfried Willhelm Leibniz Universität Hannover, 1 Welfengarten, \\ D-30167 Hannover, Germany \\ ${ }^{b}$ Laser Zentrum Hannover e.V., 8 Hollerithallee, 30419 Hannover, Germany \\ 'Institut für Gerätebau und Produktentwicklung (IPEG), Gottfried Wilhelm Leibniz Universität \\ Hannover, 1 Welfengarten, D-30167 Hannover, Germany
}

\begin{abstract}
The development of laser-based lighting systems has been the latest step towards a revolution in illumination technology brought about by solid-state lighting. Laser-activated remote phosphor systems produce white light sources with significantly higher luminance than LEDs. The weak point of such systems is often considered to be the conversion element. The high-intensity exciting laser beam in combination with the limited thermal conductivity of ceramic phosphor materials leads to thermal quenching, the phenomenon in which the emission efficiency decreases as temperature rises. For this reason, the aim of the presented study is the modeling of remote phosphor systems in order to investigate their thermal limitations and to calculate the parameters for optimizing the efficiency of such systems. The common approach to simulate remote phosphor systems utilizes a combination of different tools such as ray tracing algorithms and wave optics tools for describing the incident and converted light, whereas the modeling of the conversion process itself, i.e. photoluminescence, in most cases is circumvented by using the absorption and emission spectra of the phosphor material. In this study, we describe the processes involved in luminescence quantum-mechanically using the single-configurational-coordinate diagram as well as the Franck-Condon principle and propose a simulation model that incorporates the temperature dependence of these processes. Following an increasing awareness of climate change and environmental issues, the development of ecologically friendly lighting systems featuring low power consumption and high luminous efficiency is imperative more than ever. The better understanding of laser-based lighting systems is an important step towards that aim as they may improve on LEDs in the near future.
\end{abstract}

Keywords: lighting, laser, remote phosphor systems, modeling, temperature-dependent, photoluminescence

\section{INTRODUCTION}

There is an increasing interest in laser-based lighting systems in view of the distinct advantages they exhibit over the use of LEDs. The white light sources produced achieve significantly higher luminance that exceeds even the brightest LEDs by a factor of 2 to 5 , whereas the much smaller étendue of these optical sources increases the system's luminous flux. Their small light-emitting surface and divergence also offers great possibilities to create sharp and exact light distributions. Furthermore, the downscaling of the optics used leads to a reduction of the required resources, while the physical separation of the optical pump source and the converter material increases design flexibility and improves the system's overall thermal management. These characteristics have been utilized at some extent in applications such as automotive lighting along with imaging and display applications, where laser-based remote phosphor systems have already been integrated in various commercial multimedia projectors as well as automotive headlamps and tail lights. However, efforts must be made to improve the efficiency of such devices and the thermal limitations caused by the conversion process ${ }^{1}$, if they are to replace more conventional light sources in the future.

Most laser-based remote phosphor systems consist of a blue-emitting laser diode usually having a peak wavelength between 450 and $460 \mathrm{~nm}$ and a conversion phosphor with emission in the yellow-green spectral range. The color

*e.chatzizyrli@1zh.de; phone +49 511 2788-287; fax +49 511 2788-100; www.lzh.de

Photonics, Devices, and Systems VII, edited by Karel Fliegel, Petr Páta,

Proc. of SPIE Vol. 10603, $1060318 \cdot$ C 2017 SPIE · CCC code:

$0277-786 \mathrm{X} / 17 / \$ 18 \cdot$ doi: $10.1117 / 12.2292735$

Proc. of SPIE Vol. 10603 1060318-1 
impression is determined by the balance between these two spectral components. Although this methods produces white light sources exhibiting a wide range of Correlated Color Temperatures (CCTs), such devices often suffer from low color-rendering properties because of missing parts in the visible spectrum, in particular in the red wavelength range ${ }^{2}$. Co-doping or using blends of phosphors with different emission spectra improves on this situation but usually at the expense of device efficiency. As a result, despite significant research on new phosphor materials, yellow garnet phosphors are still predominantly used in lighting applications. They have quantum efficiencies mostly well above $90 \%$ and a very strong absorption at blue wavelengths due to an allowed $4 \mathrm{f} \rightarrow 5 \mathrm{~d}$ transition of the $\mathrm{Ce}^{3+}$ activator ion. Garnet phosphors typically show a broad yellow-green emission spectrum with a dominant wavelength of about $570 \pm 7 \mathrm{~nm}$ and outstanding chemical stability ${ }^{3}$. For these reasons, the main focus here is deriving a phosphor material model integrating the optical properties of YAG:Ce and their temperature dependence.

Modeling a remote phosphor system is a highly complex and sophisticated problem, since the scattering of the exciting and converted photons, the absorption and re-emission properties of the phosphor as well as the interaction between the optical pump source, the conversion layer and its supporting mechanical structure have to be taken into account. Simple theoretical approaches are of limited usefulness and numerical methods involving ray-tracing and wave optics algorithms as well as advanced material models have to be utilized in order to optimize the device properties. A flowchart of the model dealing with these issues along with the working principles of the phosphor conversion model are presented here.

\section{METHODOLOGY}

In some commercial ray-tracing software there are available tools for simulating phosphor-converted white light sources $^{4,5}$. These simulation techniques, albeit powerful, are limited in the sense that they disregard the temperature dependence of the optical properties of the phosphor material and are time-independent; they hold true just for a moment in time. Simulation strategies where additional tools are employed for modeling the thermal effects often have the drawback of leading to un-coupled opto-thermal simulations. Keeping the basic structure of the ray-tracing method for phosphor-converted white light sources ${ }^{6}$, we developed a time-dependent model integrating the description of the photoluminescence process itself, the phosphor conversion model. In each time step, the temperature profile of the phosphor layer is calculated based on the system's energy balance equation and its interaction with the environment (heat sinks, convection, etc.). This profile is subsequently fed to the phosphor conversion model, where the emission and absorption spectra as well as the quantum efficiency of the phosphor material are computed. By producing temperaturedependent absorption and emission spectra the thermal effects on the optical properties of the phosphor are modeled. The basic outline of the processes involved are given in the following, see also Figure 1.

Most converter materials are usually only available as powders; phosphor particles of sizes in the order of microns are immersed in an epoxy resin or silicon matrix. Evidently, for propagating light through a granular medium the knowledge of the scattering properties of this medium is essential. The scattering problem is split in two parts. The first is to solve the single-particle scattering problem in the wave optics regime, namely to calculate the extinction, absorption and scattering cross-sections of a particle. The second part is then to propagate the light incoherently, applying the scattering properties of the single particles. Rayleigh scattering fails to model this process, since the particle dimension reaches that of the light wavelength and interference effects set in. Mie theory ${ }^{7}$ which is an exact solution to Maxwell's equations is a valid approach for particle scattering in the converter powder. However, the varying particle sizes have to be taken into account by averaging the individual scattering functions, namely it is essential to know the particle size distribution as well as the material's refractive index. Since absorption is dealt separately, the imaginary part of the phosphor's complex refractive index can be ignored when dealing with a scattering event within the medium.

Considering a ray that enters the phosphor material, the average distance the ray travels without hitting a phosphor particle is determined by the mean free path length (or equivalently by the extinction coefficient). In the case of a scattering event within the boundaries of the medium, a decision has to be made of whether the scattering is elastic or inelastic. The probabilities are calculated from the absorption and scattering cross-sections. In the case of elastic scattering, the ray retains its original wavelength. The new direction of propagation, relative to the incoming direction, must be derived from the scattering function. The probability of the ray hitting non-luminescent particles having their own scattering properties, but where no absorption occurs, must also be considered. If the ray is absorbed, the quantum efficiency determines the probability of re-emission. The energy of the ray is distributed over the emission spectrum. 
Due to the Stokes shift, energy is lost and the ray is assigned a longer wavelength. The direction of propagation of the converted ray should be independent of the incoming ray and therefore isotropic in a homogeneous medium.

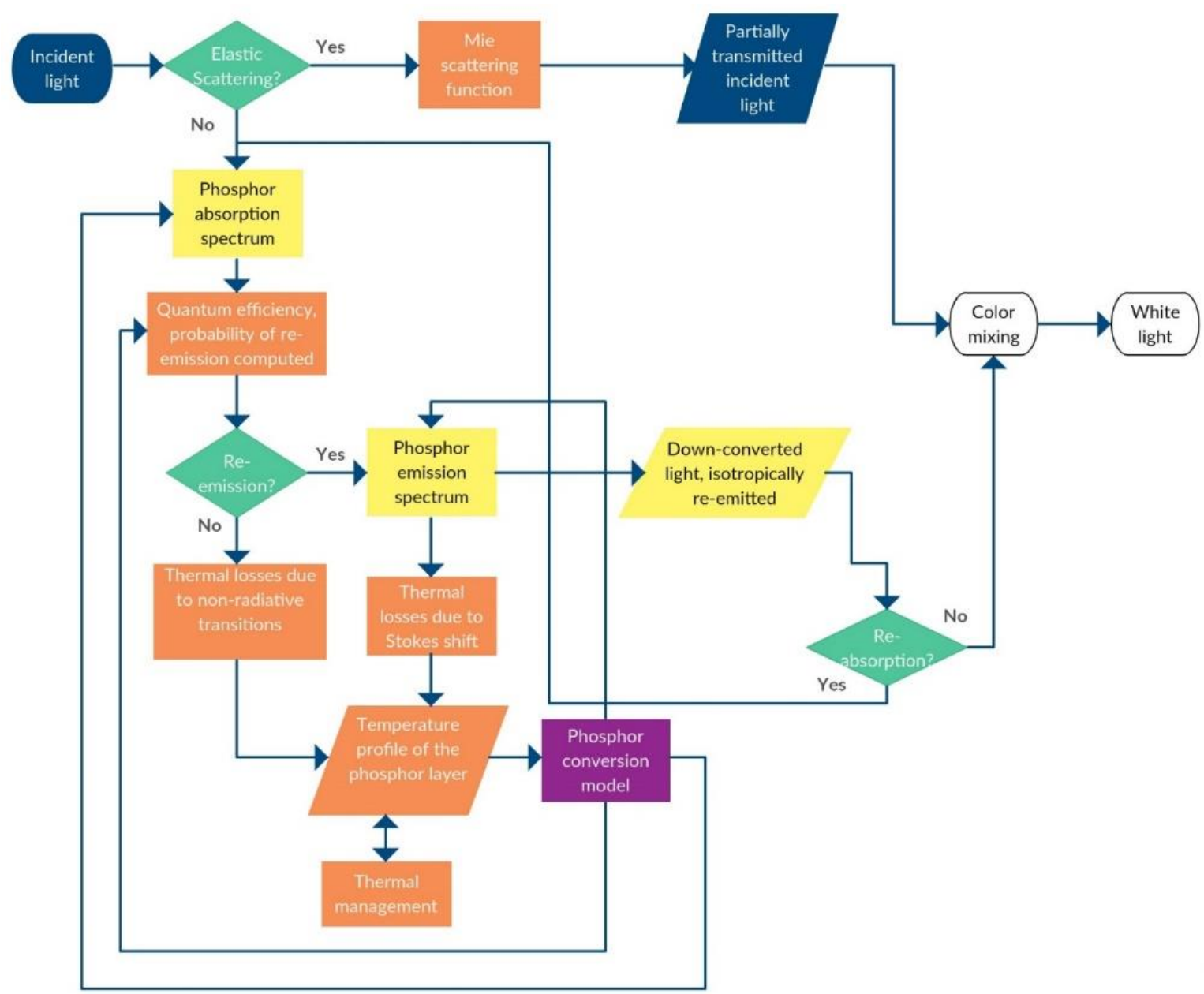

Figure 1. Simulation model schematic in the form of a simplified flowchart. An oval representing a start or end point, an arrow being a connector that shows relationships between the representative shapes, a parallelogram corresponding to the data produced, a rectangle representing a process and a diamond indicating a decision.

The thermal losses associated with Stokes shift along with the non-radiative transitions of luminescent and nonluminescent particles result in a temperature shift within the phosphor layer. The heat equation is solved in order to attain the temperature profile of the converter material. Cooling mechanisms depending on the design of the device simulated must also be taken into account. The temperature profile is subsequently fed to the phosphor conversion model, where the probabilities of the radiative and non-radiative transitions are re-calculated based on the current temperature values, namely the quantum efficiency, as well as the absorption and emission spectra of the phosphor, see Section 3. It should be noted that in most phosphors used in lighting applications there is an overlap between the emission spectra and their excitation wavelengths ${ }^{8}$. As a result, calculating the probability of re-absorption must also be included for obtaining accurate results.

The need for opto-thermal analysis rises as luminescence can be quenched with increasing temperature, a phenomenon called thermal quenching. This produces restrictions for the lighting systems developed as the device temperature (or at least the phosphor layer) must be limited below the luminescence quenching temperature. The mechanisms involved in thermal quenching heavily depend on the material used. Systematic research on the temperature dependence of the 
luminescence intensity and life time for various phosphors for lighting applications can be found in literature ${ }^{8}$. The focus here is YAG:Ce. The temperature dependence of luminescence varies both as a function of Ce-concentration and excitation wavelength. The intrinsic quenching temperature of the $\mathrm{Ce}^{3+}$ activator ion is high, well above $680 \mathrm{~K}$. The lower quenching temperatures that commercial YAG:Ce phosphors with relatively high $\mathrm{Ce}^{3+}$ concentration exhibit can be explained by thermally activated concentration quenching and is observed at temperatures above 400K. Furthermore, it is shown ${ }^{8}$ that for exciting wavelengths in the range from 450 to $490 \mathrm{~nm}$ the quenching temperature is less pronounced at longer wavelengths for the lower concentration samples. At high $\mathrm{Ce}^{3+}$ concentrations there is hardly any dependence on the excitation wavelength. From the above, the importance of the appropriate selection of the phosphor used is highlighted. Samples with smaller amounts of activator ions should be used. The loss in absorption strength can be compensated by a longer optical path way, e.g. by increasing the thickness of the YAG:Ce ceramic disk. This relationship can also be investigated by the model presented here.

Lastly, the part of the incident light transmitted through the phosphor layer along with the down-converted light emitted by the phosphor particles generate white light by color mixing. In order to evaluate the system's output the radiometric values obtained must be converted to photometric. Lighting system performance metrics such as the luminous efficacy, Color Rendering Index (CRI), Correlated Color Temperature (CCT) and etc. are calculated.

\section{PHOSPHOR CONVERSION MODEL}

When considering the optical absorption or emission within a single ion or a group of ions in a solid, it is appropriate to treat an optical transition with a localized model rather than the band theory ${ }^{9}$. Hence, the concept of the luminescent center is introduced. The simplest model of a luminescent center is the single configurational coordinate model used to describe its optical properties, particularly the effects of lattice vibrations. The configurational coordinate model explains these properties on the basis of potential curves, each of which represents the total energy of the molecule, i.e. the sum of the electron and ion energies, in its ground or excited state as a function of the configurational coordinate.

The two parabolas representing the ground $(\mathrm{u})$ and excited (v) states are displaced along one vibrational coordinate $\mathrm{x}$, the ground state minimum being at $\mathrm{x}=0$ and the upper state minimum at $\mathrm{x}=\mathrm{a}$. Natural measures of the coordinate offset between the two parabolas are the energies $S_{u} \hbar \omega_{u}$ and $S_{v} \hbar \omega_{v}$, which are the energy differences between the two minima $x$ values in units of the phonon energies of the parabolas. The two parabolas are also displaced in the energy coordinate, a reasonable offset being the zero-phonon energy $h v_{z p, v u} \equiv E_{z p, v u}$. In general, the following equations apply for the curves in Figure 2:

and

$$
E_{v}=E_{z p, v u}-\frac{1}{2}\left(\hbar \omega_{v}-\omega_{u}\right)+S_{v} \hbar \omega_{v}\left(\frac{x}{\alpha}-1\right)^{2}
$$

$$
E_{u}=S_{u} \hbar \omega_{u}\left(\frac{x}{\alpha}\right)^{2}
$$

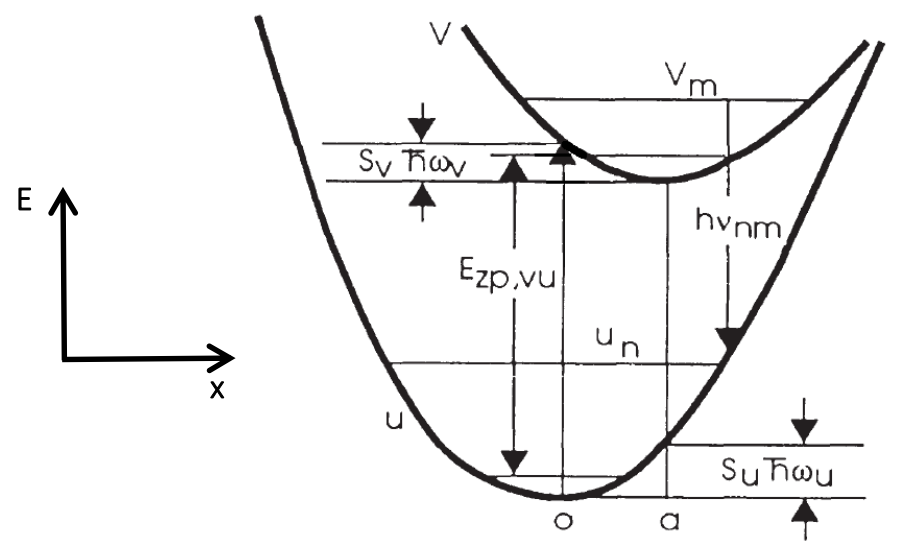

Figure 2. The single configurational model ${ }^{10}$. 
In Figure 2, the optical absorption and emission processes are indicated by the single vertical arrows. Neglecting small corrections due to differences in zero-point energies, there will be absorption centered at $h v_{\mathrm{abs}}=h v_{\mathrm{zp}, \mathrm{vu}}+\mathrm{S}_{\mathrm{v}} \hbar \omega_{\mathrm{v}}$ and emission centered at $h v_{\text {emis }}=h v_{\mathrm{abs}}-\mathrm{S}_{\mathrm{u}} \hbar \omega_{\mathrm{u}}-\mathrm{S}_{\mathrm{v}} \hbar \omega_{\mathrm{v}}$. As the illustration shows, the nucleus of an emitting ion stays approximately at the same position throughout the optical processes. This is called the Franck Condon principle ${ }^{9}$. This approximation is quite reasonable since an atomic nucleus is heavier than an electron by $10^{3}$ to $10^{5}$ times. Furthermore, the spatial distribution of an electron is different between the ground and excited states resulting to a difference in the electron wavefunction overlap with neighboring ions. This difference further induces a change in the equilibrium position and the force constant of the ground and excited states and is the origin of the Stoke's shift.

A simple model such as this can qualitatively explain Stoke's law, the widths of absorption or emission bands and their temperature dependence as well as the thermal quenching of luminescence. The study of thermal quenching in phosphors is of particular interest since it imposes limitations to the efficiency of phosphor-converted lighting devices. Although a multidimensional configurational model can give a quantitatively valid explanation of thermal quenching in phosphors, C.W. Struck and W.H. Fonger ${ }^{10}$ have shown that in practice their proposed $\mathrm{W}_{\mathrm{p}}$ functions derived from the single configurational model can adequately describe the processes involved. The phosphor conversion model integrated to the ray-tracing scheme discussed in Section 2 uses these functions to model the temperature-dependent behavior of phosphors used in lighting applications and in particular YAG:Ce ${ }^{11}$.

For a brief description of the working principles of the model, the special case where both ground and excited states have the same angular frequency is considered, i.e. the equal force constants model. In this case, following the notation of Figure 2, the equalities $\hbar \omega_{\mathrm{u}}=\hbar \omega_{\mathrm{v}}$ and $S_{\mathrm{u}}=\mathrm{S}_{\mathrm{v}} \equiv \mathrm{S}_{0}$ apply.

For the simple equal-force constants luminescent center, supposing that the ground state vibrational wavefunction is $u_{n}$ and the offset state wavefunction is $\mathrm{v}_{\mathrm{m}}$, the bandshapes are given as a distribution over $\mathrm{p}$, the number of phonons of energy $\hbar \omega$ created by the transition having photon energy $h v_{v u, p}$ in absorption and $h v_{u v, p}$ in emission, according to

$$
\mathrm{p} \hbar \omega \equiv(\mathrm{m}-\mathrm{n}) \hbar \omega=\mathrm{h} v_{\mathrm{vu}, \mathrm{p}}-\mathrm{h} v_{\mathrm{zp}, \mathrm{vu}} \quad \text { in absorption }
$$

and

$$
\mathrm{p} \hbar \omega \equiv(\mathrm{n}-\mathrm{m}) \hbar \omega=\mathrm{h} v_{\mathrm{zp}, \mathrm{vu}}-\mathrm{h} v_{\mathrm{uv}, \mathrm{p}} \quad \text { in emission. }
$$

All transitions $u_{n} \rightarrow v_{m}$ in absorption and $v_{m} \rightarrow u_{n}$ in emission with their indices following Equations (3) and (4) share a common photon energy. The $p$ index runs from $-\infty$ to $\infty$, with a positive value implying creating phonons. The maximum of this distribution will be near $\mathrm{p}=\mathrm{S}_{0}$.

The normalized bandshapes are given by Boltzmann-weighted Frank-Condon factors, namely squared overlap integrals. These shape functions are:

$$
\mathrm{W}_{\mathrm{p}}=\sum_{\mathrm{n}=\max (0,-\mathrm{p})}^{\infty}(1-\mathrm{r}) \mathrm{r}^{\mathrm{n}}\left|\left\langle\mathrm{v}_{\mathrm{p}+\mathrm{n}} \mid \mathrm{u}_{\mathrm{n}}\right\rangle\right|^{2} \quad \text { in absorption }
$$

and

$$
\mathrm{W}_{\mathrm{p}}=\sum_{\mathrm{m}=\max (0,-\mathrm{p})}^{\infty}(1-\mathrm{r}) \mathrm{r}^{\mathrm{m}}\left|\left\langle\mathrm{u}_{\mathrm{p}+\mathrm{m}} \mid \mathrm{v}_{\mathrm{m}}\right\rangle\right|^{2} \quad \text { in emission }
$$

where $r=\exp (-\hbar \omega / \mathrm{kT})$ is the Boltzmann factor.

As for a measure of the non-radiative transitions probability, one can consider the crossover energy, $\mathrm{E}_{\mathrm{c}}$. When the two configurational coordinate curves intersect with each other, an electron in the excited state can cross the intersection assisted by thermal energy and reach the ground state non-radiatively. Here, the two parabolas have their crossover at:

$$
\frac{\mathrm{x}_{\mathrm{c}}}{\alpha}=\frac{1}{2}\left(\frac{\mathrm{E}_{\mathrm{zp}, \mathrm{vu}}+\mathrm{S}_{0} \hbar \omega}{\mathrm{S}_{0} \hbar \omega}\right)^{2}
$$

and

$$
E_{c}=\frac{\left(E_{z p, v u}+S_{0} \hbar \omega\right)^{2}}{4 S_{0} \hbar \omega}
$$


Although, the non-radiative rate of the transition $\mathrm{v} \rightarrow \mathrm{u}$ from a vibrational state $\mathrm{m}$ to the final state $\mathrm{n}=\mathrm{p}+\mathrm{m}$ is expected to be proportional to the Boltzmann factor for the availability of the crossover, it is shown ${ }^{10}$ that the same sum of thermally weighted Franck-Condon factors,

$$
\mathrm{R}_{\mathrm{uv}, \mathrm{p}}=\mathrm{A}_{\mathrm{uv}} \mathrm{W}_{\mathrm{p}}
$$

with $\mathrm{W}_{\mathrm{p}}$ as in Equations (5) and (6) and $\mathrm{A}_{\mathrm{uv}} \approx 10^{12}-10^{14}$, will give a very reasonable picture of the non-radiative transition rate, where $\mathrm{p}$ is determined via

$$
\mathrm{p} \hbar \omega=\mathrm{h} v_{\mathrm{zp}, \mathrm{vu}}
$$

which is the analogue of Equations (3) and (4) for zero photon energy.

\section{CONCLUSIONS}

Laser-based remote phosphor systems are the next stepping stone in the solid-state lighting technology. They are excellent candidates for replacing LEDs and more conventional light sources in applications where high luminance is required. However, the resulting devices have still performance issues that are traced amongst other sources in the conversion process within the phosphor layer. The high-intensity exciting laser beam in combination with the limited thermal conductivity of ceramic phosphor materials leads to the phenomenon of thermal quenching. Therefore, it is essential to develop appropriate tools that investigate the thermal limitations of these devices in the interest of designing an optical system that would work with optimal efficiency, namely on the thermal limit of the phosphor layer.

Modeling the processes involved in the generation of a phosphor-converted white light source is a complex and sophisticated problem. The time-dependent opto-thermal model proposed here addresses the scattering problem of the exciting and converted light as well as the temperature dependence of the absorption and emission characteristics of the phosphor material. In particular, the photoluminescence phenomenon and its temperature dependence is treated by the phosphor conversion model. Lastly, another issue addressed here is tuning the parameters of the cooling mechanisms implemented for sustaining a specific temperature within the phosphor layer. The study of the thermal management system and its influence on the overall device efficiency gives rise to the need of time-dependent simulations.

\section{ACKNOWLEDGEMENTS}

The PhD program Tailored Light is funded by the Lower Saxony Ministry for Science and Culture (MWK), Germany.

\section{REFERENCES}

[1] Lenef A., Kelso J., Zheng Y. and Tchoul M., "Radiance limits of ceramic phosphors under high excitation fluxes", Proc. SPIE 8841, Current Developments in Lens Design and Optical Engineering XIV, 884107 (2013)

[2] Basu C., Kloppenburg G., Wolf A., Wollweber M., Roth B. and Lachmayer R., "A high power blue diode laser pumped white light source using remote phosphors.”, Proc. ISAL, (2013)

[3] Xie R. J., Li Y. Q., Hirosaki N. and Yamamoto H., [Nitride phosphors and solid-state lighting], Taylor \& Francis Group, CRC Press, USA, 79-110 (2011)

[4] Zollers M., "Phosphor modeling in LightTools: Ensuring accurate white LED models", White paper, <https://optics.synopsys.com/lighttools/pdfs/ModelingPhosphorsInLightTools.pdf>, (2011)

[5] Gangadhara S., "Modeling a white light source using the phosphor DLL", <http://www.zemax.com/os/ resources/learn/knowledgebase/modeling-a-white-light-source-using-the-phosphor-d>, (2011)

[6] Linder N., Eisert D., Jermann F. and Berben D., "Simulation of LEDs with phosphorescent media for the generation of white light", [Nitride semiconductor devices: principles and simulation], Wiley-VHC Verlag GmbH \& Co, Germany, 327-351 (2007)

[7] Bohren C. F. and Huffmann D.R., [Absorption and scattering of light by small particles], Wiley-Interscience, USA, (1983) 
[8] Bachmann V., [Studies on luminescence and quenching mechanics in phosphors for light emitting diodes], Doctoral thesis, Datawyse B.V., Netherlands, (2007)

[9] Yen W., Shionoya S. and Yamamoto H., [Phosphor handbook], Taylor \& Francis Group, CRC Press, USA, 5972, 163-178, 215-238, 557-576 (2007)

[10] Struck C.W. and Fonger W.H., [Understanding luminescence spectra and efficiency using Wp and related functions], Springer-Verlag Berlin Heidelberg, Germany, (1991)

[11] Bachmann V., Ronda C. and Meijerink A., "Temperature quenching of yellow $\mathrm{Ce}^{3+}$ luminescence in YAG:Ce", Chem. Mater. 21(10), 2077-2084 (2009) 